\title{
Le Retour de Judith à Béthulie de Botticelli
}

\section{Céline Coussy}

\section{(2) OpenEdition}

Journals

Édition électronique

URL : http://journals.openedition.org/clio/9466

DOI : 10.4000/clio.9466

ISSN : 1777-5299

Éditeur

Belin

Édition imprimée

Date de publication : 15 décembre 2009

Pagination : 181-194

ISSN : 1252-7017

\section{Référence électronique}

Céline Coussy, «Le Retour de Judith à Béthulie de Botticelli », Clio. Histoire, femmes et sociétés [En ligne], 30 | 2009, mis en ligne le 15 décembre 2012, consulté le 19 avril 2019. URL : http:// journals.openedition.org/clio/9466 ; DOI : 10.4000/clio.9466 


\section{Document}

\section{Le Retour de Judith à Béthulie de Botticelli}

Céline Coussy

Sandro Botticelli a choisi de peindre, au début de sa carrière, un épisode de l'Ancien Testament, mettant en scène une femme à la fois très populaire au Moyen Âge et à Florence : Judith.

Cet épisode de l'Ancien Testament ${ }^{1}$ narre la résistance de la ville juive de Béthulie face au général assyrien, Holopherne. Une belle veuve de Béthulie, Judith, décide de se rendre dans le camp ennemi. La jeune femme, avec l'aide de sa servante, Abra, se pare de ses plus beaux atours. Holopherne est séduit par sa beauté. Après un festin très arrosé, Judith, restée seule avec le général dans sa tente, le décapite. Elle cache sa tête sanglante dans un sac, et revient dans la nuit, avec sa servante, à Béthulie. La veuve explique alors son exploit et conjure son peuple d'attaquer l'armée assyrienne dès l'aube. Cette dernière, surprise par l'assaut, cherche son général, mais ne découvre qu'un corps sans tête. Les soldats, privés de commandement, sont massacrés par les Hébreux.

Ce récit biblique de l'exploit de Judith connaît une grande popularité au Moyen Âge. Poèmes, récits, en latin ou en langue vernaculaire, enluminures, vitraux, sculptures, montrent que cette figure est très présente tout au long du Moyen Âge.

1 Bible de Jérusalem 1998 : Livre de Judith : 858-882. 
Botticelli a peint sur deux panneaux les épisodes qui ont suivi la décapitation d'Holopherne par Judith. Le Retour de Judith à Béthulie et la Découverte du corps d'Holopherne sont conservés et exposés ensemble au Musée des Offices de Florence. Leur datation reste discutée (vers 1469) et leur commanditaire inconnu. Par contre, il n'existe aucun doute sur leur auteur : Alessandro Botticelli. À cette époque, le jeune peintre vient de quitter son maitre, Filippo Lippi et il commence une carrière d'artiste réputé à Florence. Pour Jaynie Anderson ${ }^{2}$, ces œuvres de jeunesse seraient des panneaux de coffre de mariage (cassone), ce que réfute Ronald Lightbown arguant de leur petitesse (31 $\mathrm{cm}$ de haut pour $25 \mathrm{~cm}$ de large). Ils appartiendraient plutôt à la catégorie des petits tableaux précieux, souvent conservés dans des coffrets en bois ou en cuir. Peut-être même formaient-ils un unique panneau, partagé depuis. Selon Lightbown, Le Retour de Judith à Béthulie et La découverte du corps d'Holopherne ont été réalisés après une Adoration des mages (1465-1467), sans doute la plus ancienne œuvre conservée de Botticelli3 , et avant La Force commandée en 1470 par la Commune de Florence.

\section{Une mise en scène à la fois traditionnelle et moderne}

Contrairement à la scène traditionnelle du meurtre d'Holopherne, climax de l'histoire, Botticelli choisit le retour à Béthulie. Ce tableau ${ }^{4}$ montre toutefois, à l'arrière-plan, une autre scène, celle de l'attaque du camp des Assyriens. À partir du XVe siècle, Judith est surtout représentée tenant une épée et la tête d'Holopherne. Botticelli se situe par son choix iconographique et sa mise en scène, entre Moyen Âge et Renaissance ${ }^{5}$. Il suit l'histoire biblique et son interprétation traditionnelle et livre ici une œuvre empruntant beaucoup à la tradition symbolique et de mise en scène médiévale, l'esthétique appartenant davantage au style de la Renaissance.

\footnotetext{
Anderson $1997: 30$.

Lightbown 1989 : 26.

Voir Cabier d'images, figure 5.

5 Les inventaires des œuvres de Botticelli cités par Lightbown ne font mention d'aucune représentation par l'artiste du meurtre avant 1495. Lightbown 1989: 359-360.
} 
Judith, luxueusement vêtue, rentre avec Abra à Béthulie, dont les remparts se dressent au fond à droite. La servante porte sur la tête celle d'Holopherne. Le sac largement entrouvert laisse voir le visage marqué par l'âge du général, les cheveux et la barbe grisonnants. Elles empruntent un chemin élevé surplombant la scène de bataille.

Botticelli situe son histoire en plein jour, contrairement au texte biblique ; peut-être pour mieux utiliser la lumière afin de sculpter ses personnages et donner une relative cohérence à l'épisode de fond.

Judith tient dans la main droite un cimeterre. Son épée est celle qu'elle a prise à Holopherne pour lui trancher la tête. Selon le texte biblique, Judith a laissé l'épée dans la tente. Pourquoi Botticelli l'a-t-il conservée ? L'épée montre le chemin suivi par les deux jeunes femmes. Elle est aussi l'attribut identifiant de l'héroïne. Le rameau d'olivier, tenu délicatement de la main gauche, symbolise la victoire et la paix. Ce signe est fréquemment utilisé dans les cérémonies publiques florentines ${ }^{6}$. À l'arrière-plan, sur les remparts de la cité de Béthulie, au bout d'un mât, est attaché soit l'étendard de la ville, soit, comme dans le texte biblique, la tête d'Holopherne. Les Juifs assiégés sortent de la ville par un pont pour attaquer les cavaliers et les fantassins assyriens. L'armée ennemie fuit, désemparée. Botticelli réunit dans un même décor deux scènes distinctes du Livre de Judith. C'est le premier exemple de narration simultanée dans son art. Ce procédé est fréquent au Moyen Âge, mais commence à être abandonné au XVIe siècle.

Judith est la figure principale du tableau : son pied droit a franchi vers l'avant la ligne centrale. La servante Abra, plus petite, courant presque derrière sa maîtresse, accentue ce mouvement. Botticelli hiérarchise ses personnages de manière traditionnelle par leur différence de taille. Il introduit toutefois un topos qui va croissant au $\mathrm{XVI}^{\mathrm{e}}$ siècle jusqu'à devenir un cliché à partir du XVII ${ }^{\mathrm{e}}$ siècle: la servante porte des vêtements moins riches et son visage est grossier (gros nez, menton très en avant).

6 Catalogue de l'exposition Botticelli, De Laurent le Magnifique à Savonarole 2003 : 98. 


\section{Les vertus de l'héroïne}

Depuis le haut Moyen Âge, Judith symbolise un certain nombre de vertus, dont les plus importantes sont l'Humilité, la Force et la Justice. Botticelli reprend ici l'interprétation médiévale: Judith apparait d'abord comme une héroïne vertueuse et morale.

Judith est en effet le plus souvent associée à l'Humilité, par exemple dans l'illustration du Speculum Virginum, un manuscrit du XII siècle, destiné aux nonnes. Humilité est présentée casquée, portant lance et bouclier, dans son combat allégorique contre Orgueil illustrant la Psychomachia de Prudence. Le vice tombe en arrière, foulé au pied par Humilité qui lui enfonce dans le sein droit sa longue épée 7 . À ses côtés, sont figurées $\mathrm{Yael}^{8}$ et Judith, tenant d'une main leur attribut (maillet ou épée), et de l'autre, une palme, symbole de victoire sur leur ennemi et sur le vice. Chacune des femmes se tient presque de face, légèrement tournée vers Humilité, et marchant sur son ennemi allongé sur le côté?.

Très tôt, les exégètes associent Judith à l'humilité. La veuve, entièrement soumise à Dieu, exécute ses ordres. Ses ornements sont interprétés comme des armures contre les assauts du vice dans le camp assyrien ${ }^{10}$. L'humilité de Judith se traduit par le recouvrement de ses cheveux par un voile ${ }^{11}$.

Donatello réalise pour Cosme ou Pierre de Médicis un bronze représentant Judith et Holopherne, vers les années 1455-1460; il se trouvait peut-être dans la cour ou le jardin du palais de la Via Larga ${ }^{12}$. Un article d'Edward Wind mentionne l'inscription originelle gravée sur son socle : Le luxe abat les royaumes, les vertus exaltent les cités / Vois comme Humilité de cette main a tranché Orgueil de ce cou ${ }^{13}$. Judith est d'abord

7 Voir Cabier d'images, figure 6.

8 Héroïne du Livre des Juges, dont l'histoire se rapproche de celle de Judith. Livres des Juges : 4-5.

9 Friedman $1986: 234$

10 Migne 1878-1891: CLXXV, 747.

11 Migne 1878-1891: CIX, 585.

12 Blake Mc Ham $2001: 33$.

13 Wind 1985 : 38. «Regna cadunt luxu surgunt virtutibus urbes / Caesa vides Humili colla Superba manu». 
une figure de l'Humilité, alors qu'Holopherne est clairement associé à la luxure. Toutefois, la scène où Judith tue Holopherne est aussi utilisée comme une allégorie de la victoire de la Tempérance sur la débauche $^{14}$ sexuelle ou l'ivresse. Le socle de la statue de Judith de Donatello est orné de trois bas-reliefs évoquant des scènes bacchiques. La tempérance de Judith a été saluée et louée très tôt. Saint Ambroise a particulièrement insisté sur la tempérance et la sobriété de Judith comme une donnée primordiale dans sa victoire contre Holopherne. Les jeûnes de la veuve ont une grande part dans sa victoire ${ }^{15}$.

Le bronze de Donatello devait être la plus célèbre représentation de l'héroïne de la ville de Florence au XVe siècle. Son interprétation théologique, morale et politique est explicitée par les liens entre la statue, le socle et les diverses inscriptions. Donatello a représenté la veuve, entièrement couverte, humble s'apprêtant à tuer un Holopherne échevelé, dénudé et ivre. Elle marque la victoire de l'humilité, de la tempérance et de la chasteté face à l'orgueil, la luxure et l'excès.

Botticelli connaissait très probablement l'œuvre de Donatello. Il a choisi lui aussi de figurer Judith en héroïne morale et chrétienne. Mais sa présentation diffère. Judith porte des habits plus élégants, sa tête n'est ornée que d'un diadème. Elle affecte une certaine coquetterie évoquée par le texte biblique ${ }^{16}$. Mais pour renforcer l'aspect chaste de son héroïne, le peintre choisit de ne pas représenter la scène préférée des artistes. Toute proximité physique entre la belle veuve et le général assyrien est ici exclue: c'est Abra qui porte la tête d'Holopherne. En choisissant de dissocier Le Retour à Béthulie de La Déconverte du corps d'Holopherne, il éloigne le spectateur de l'intimité de la chambre, là où la chasteté de la veuve a été mise en péril. Cette distanciation spatiale et temporelle adoucit l'aspect quelque peu subversif d'une scène de séduction et de meurtre, de plus en plus détournée à l'aube de la Renaissance par d'autres artistes qui soulignent la perfidie de la Femme. L'aspect séducteur de Judith est

\footnotetext{
14 Friedman $1986: 235$.

15 Migne 1878-1891 : XVI, 247.s

16 Livre de Judith : 10, 1-5.
} 
tempéré : ses vêtements couvrent l'essentiel de son corps, ses bijoux restent discrets ${ }^{17}$. Jolie, féminine, élancée, elle n'est ni sensuelle ni sexualisée. Botticelli choisit de montrer une guerrière victorieuse et chaste. Cette chasteté tient une place prépondérante dans la place accordée à l'honneur pour les femmes du Moyen Âge ${ }^{18}$.

Le héros et l'héroïne se caractérisent par leur grand courage, leur force d'âme dans des situations exceptionnelles, devant le danger et face à la douleur physique et morale. Ainsi, ces vertus sont une condition primordiale à l'accès au statut de héros ${ }^{19}$. Judith est célébrée très tôt dans le monde chrétien pour son courage. Raban Maur et saint Ambroise louent Judith qui puise sa force dans sa foi en Dieu ${ }^{20}$. L'association iconographique Judith/Force n'apparait pas avant le $\mathrm{XIV}^{\mathrm{e}}$ siècle, et dans un seul ouvrage : Chansonne des Sept Vertus et Sept Arts libéraux, enluminée par Bartolomeo di Bartoli21. La veuve y est associée à un homme tuant un lion et légendé Fortitudo. Ce personnage masculin évoque d'autres tueurs de lions, tels Samson et Hercule ; par ailleurs, cet homme semble porter une couronne, ce qui le rapprocherait d'une autre figure biblique : David ${ }^{22}$. Dès la seconde moitié du XIII siècle, Hercule est devenu une incarnation de la Force, en tant que vertu chrétienne. C'est au XIV e siècle, en Italie surtout, que la figure du héros mythologique est associée à celle de David et de Samson. Déjà, Dante compare David à Hercule comme l'exemple du combattant qui devient fort grâce à l'aide de Dieu ${ }^{23}$.

Un peu plus d'un siècle plus tard, Botticelli semble suivre cette association comme le laisse penser la ressemblance du visage mélancolique de la Force avec celui de Judith dans le Retour de Judith à

\footnotetext{
17 Burzlaff $2006: 21$.

18 Gauvard $2001: 163$.

19 Contamine 1999 : 407.

20 Migne 1878-1891: CIX, 563.

21 L'Index of Cristian Art de l'Université de Princeton en indique une référence au Musée national d'art antique à Rome et datant du XIVe siècle. Le Musée Condé à Chantilly en possède un autre exemplaire, dont le folio $3 \mathrm{R}$ est inscrit dans la base Joconde. Ce manuscrit a été fait à Milan avant 1349.

22 Smith $1995: 72$.

23 Smith $1995: 71$.
} 
Béthulie $^{24}$. Judith est une figure traditionnelle de la Force. Son courage est d'autant plus important que la prouesse individuelle est hautement mise en valeur au Moyen Âge ${ }^{25}$. Judith agit bien seule contre tous $^{26}$ : contre les habitants désespérés de Béthulie et contre l'armée assyrienne menaçante. Cette force morale est d'autant plus frappante que les héros sont physiquement faibles. En effet, Dieu frappe les forts par les humbles et les faibles ${ }^{27}$. Aussi la figure de Judith est-elle très souvent associée à celle de David, tuant le géant Goliath, particulièrement à la Renaissance ${ }^{28}$.

Judith symbolise donc deux vertus essentielles, dans un temps où politique et vertus sont liées. En cette fin du XVe siècle à Florence, certains appellent au redressement politique par le redressement moral.

\section{Une image politique à Florence}

L'aspect politique de ce tableau n'a pas fait l'objet de beaucoup de commentaires. L'anonymat de son commanditaire explique cette relative absence par comparaison avec les œuvres plus explicitement politiques de la fin de la carrière du peintre. Toutefois, l'importance de Judith à Florence et la proximité de Botticelli avec les Médicis laissent une place à une interprétation politique du panneau ${ }^{29}$.

Une autre vertu morale et politique dont le poids va croissant au Moyen Âge est la Justice. Au XIII ${ }^{\mathrm{e}}$ siècle, certains théologiens, notamment Thomas d'Aquin, n'évoquent plus l'Humilité comme la reine des vertus, ils lui préfèrent la Charité ou la Justice qui doivent régir la relation entre Dieu et les hommes ${ }^{30}$. Il s'agit de défendre le système social et politique au travers de la thématique du Bien Commun. Pour conserver cet ordre social, la Justice devient une arme

\footnotetext{
24 Catalogue de l'exposition Botticelli, De Laurent le Magnifique à Savonarole 2003 : 98.

25 Contamine 1995 : 411.

26 Abra ne faisant que suivre sa maitresse dans le texte.

27 Migne 1878-1891: CIX, 563, CLXXV : 744-745.

28 Bialostocky 1981: 121.

29 Burzlaff $2006: 20$.

30 Casagrande Vecchio 2003 : 43.
} 
nécessaire et indispensable ${ }^{31}$. L'histoire de Judith renvoie, tel un exemplum, à l'importance d'une justice divine dont les arrêts sont exécutés par les hommes. D'ailleurs, en Italie, Judith (Giuditta) se voit rapprocher phonétiquement de la Justice (Justicia). Judith représente une justice punitive, un «bras séculier ». Son épée s'abat sur ceux qui menacent le Bien Commun. Elle est le bras armé du seul juge reconnu par la religion chrétienne : Dieu ${ }^{32}$.

Judith apparaît donc comme l'héroïne qui a permis la défense et la survie de son peuple. Cette conception est très présente dans les mentalités juives: Judith est associée aux autres grands héros libérateurs, Judas Macchabée ou Samson ${ }^{33}$. Elle s'inscrit ainsi dans l'histoire troublée de l'Italie communale de la fin du XVe siècle où la question de la légitimité du pouvoir et de la tyrannie est centrale ${ }^{34}$.

Jusqu'au XII e siècle, les clercs se sont surtout préoccupés de prôner l'obéissance au prince, tout en définissant les devoirs de celuici envers ses sujets. Ainsi, les théoriciens s'appuient sur l'idée de pacte entre le prince et son peuple ${ }^{35}$. Au XII ${ }^{\text {e }}$ siècle, les idées se précisent quant au droit de désobéissance. Jean de Salisbury (1115-1180) apporte au Moyen Âge l'une des réflexions les plus influentes sur le tyrannicide dans son Policraticus. Selon lui, le prince, en se comportant comme un tyran, délie la population de son pacte, et donc de son obéissance $^{36}$. Tuer le tyran relève donc de l'acte légitime et juste. Jean de Salisbury émet quand même des conditions au tyrannicide : il ne faut y recourir qu'en dernier recours ${ }^{37}$. Il précise aussi que le meilleur moyen de mettre à bas la tyrannie est de recourir à Dieu par la prière $^{38}$. Le théologien cite Judith comme le plus important exemple biblique de tyrannicide ayant sauvé le peuple juif ${ }^{39}$. L'histoire de

\footnotetext{
31 Edgerton 1972 : 78 : Thomas d'Aquin préconise la peine de mort pour maintenir le Bien Commun.

32 Edgerton 1972: 69.

33 Friedman $1986: 231$.

34 Turchetti $2001: 217$.

35 Turchetti $2001: 241$.

36 Turchetti $2001: 247$.

37 Turchetti $2001: 255$.

38 Coville 1932 : 192.

39 Blake Mc Ham 2001 : 40.
} 
Judith fournit donc aux auteurs et aux artistes un prétexte à l'illustration du tyrannicide et le Policraticus tient une place fondamentale dans la pensée politique italienne.

Parmi les cités italiennes, c'est sans doute Florence qui développe le plus le thème de Judith, au XVe siècle, dans la littérature et les arts. Pétrarque et Boccace se sont penchés sur la tyrannie et le tyrannicide. En 1400, le chancelier de Florence, Coluccio Salutati poursuit ce débat avec son De tyranno, où il reprend Jean de Salisbury. Au début $\mathrm{du} \mathrm{XV}^{\mathrm{e}}$ siècle, Florence est en guerre contre le Milan des Visconti. Leonardo Bruni, chancelier de la république florentine, présente cet affrontement comme celui des libertés républicaines face à la tyrannie seigneuriale. Florence se présente donc comme le principal défenseur de la République et justifie ainsi, au passage, sa politique expansionniste.

Judith, depuis longtemps associée à la République et principale figure du tyrannicide, est donc reprise par la capitale toscane. Durant la première moitié du XVe siècle, Florence voit s'ériger un grand nombre d'œuvres symbolisant les responsabilités civiques et les vertus républicaines ${ }^{40}$. En 1401, Lorenzo Ghiberti insère dans une petite niche des portes du Baptistère, une figure de Judith triomphante, l'épée levée et tenant la tête d'Holopherne. Certains y voient une référence politique, selon le modèle iconographique de la Justice: Dieu intervenant en faveur de la République. Frank Capozzi en doute fortement : la Judith de Ghiberti s'insèrerait plutôt dans un registre religieux ${ }^{41}$.

Cette signification politique de Judith est plus clairement exprimée avec l'ascension des Médicis vers le pouvoir. Après sa victoire sur les Albizzi, Cosme reste fidèle au régime républicain, tout en confiant à ses proches des postes clés. Le sort de la statue de Judith de Donatello correspondrait à cette prise de pouvoir progressive. La première inscription semble indiquer que Judith représente les vertus citoyennes, dont l'humilité ${ }^{2}$. La famille Médicis, en commandant cette statue, en relation avec le David de Donatello, autre symbole du

\footnotetext{
40 Capozzi $1975: 25$.

41 Capozzi $1975: 42$.

42 Wind 1985 : 38. Voir plus haut.
} 
tyrannicide, se poserait en protectrice des libertés de la ville. Pierre renforce ce message. Entre 1464 et 1466, il fait graver sur le socle de la statue :

Le salut de l'Etat. Pierre de Médicis, fils de Cosme dédicace cette statue d'une femme à la liberté et à la force [morale], avec lesquelles les citoyens invaincus et aux cœurs constants retourneront dans la République ${ }^{43}$.

Cette seconde inscription glorifierait les vertus civiques et la victoire de Pierre sur une conspiration menée par Luca Pitti ${ }^{44}$. Pierre se veut encore le défenseur de la République, à l'image de Judith. Placées originellement dans le palais des Médicis, ces deux statues montrent le rejet officiel et ostensible par la famille de toute velléité tyrannique. Judith serait donc le symbole médicéen du respect de la République ${ }^{45}$.

Botticelli aurait, quant à lui, souhaité mettre l'accent sur l'héroïne salvatrice de son peuple ${ }^{46}$. Il souligne son rôle civique par l'épée qui pointe vers l'olivier, symbole de paix. Botticelli signifierait-il que la paix n'est possible que par une défense armée ? Toujours est-il que sa Judith retourne auprès de sa communauté. Le texte biblique précise que Judith n'a jamais souhaité aucune responsabilité politique malgré son exploit, retournant à son veuvage solitaire ${ }^{47}$. Elle n'aurait agi que pour le bien de sa ville.

Quand en 1434, Cosme de Médicis prend le contrôle de Florence, son ascension politique se fait au détriment des libertés républicaines. Mais la ville semble y gagner en stabilité. Le pouvoir des Médicis connait des revers de fortune, mais reste fort vers 1470. Donatello aurait ainsi représenté la prise de pouvoir et le désir de protection de la république par la nouvelle puissante famille. Botticelli aurait souligné l'effet bénéfique de ce patronage : Béthulie devient Florence,

43 Capozzi : 27. «Salus Publica. Pietro Medices Cos. fi. Libertati simul et fortitudini hanc mulieris statuam quo cives invicto constantique animo ad rem pub. redderent dedicavit ».

44 Capozzi $1975: 28$.

45 Blake Mc Ham 2001 : 41 et 44. «Victor est quisquis patriam tetur./Frangit immamis Deus hostis iras./En puer grandem domuit tiramnum./Vincite, civite!»

46 Burzlaff $2006: 22$.

47 Livre de Judith : 16, 21-25. 
et Judith, une représentante de la famille des Médicis. Le rameau d'olivier tenu par Judith symboliserait la victoire et la paix qu'apportent les Médicis ${ }^{48}$. Mais la petite taille des panneaux de Botticelli semble interdire toute volonté de propagande publique, contrairement à la statue de Donatello.

Les liens entre Botticelli et la famille Médicis sont encore difficiles à établir au début du parcours du peintre, même si de fortes présomptions existent en leur faveur. C'est à l'occasion de la réalisation de la Force, que l'historien commence à être mieux documenté. En 1469, un notable, proche de Pierre de Médicis, Tommaso Soderini, devient un mécène de Botticelli. Sandro semble obtenir assez vite les faveurs de l'influente famille. Vasari indique que, parmi ses premiers commanditaires, on compte Laurent le Magnifique. Lightbown en doute, préférant un mécénat par des proches. Ce n'est qu'en 1475 qu'est attestée la première commande de Julien, frère de Laurent le Magnifique, à Botticelli. Dans sa jeunesse, le peintre est en contact avec les Médicis, mais surtout avec des branches voisines ou cadettes, ou des amis proches comme Tommaso. Ces tableaux, Le Retour de Judith à Béthulie et La Découverte du corps d'Holopherne, gages de fidélité, seraient destinés à des partisans de la riche famille, mais pas forcément à être connus de tous.

Peindre Judith, pour un artiste florentin, ne relève pas du hasard. Elle est l'un des personnages féminins vétérotestamentaires les plus populaires. Elle est aussi la libératrice d'un peuple, symbole d'autant plus important à une époque où les frontières du contado restent mal définies et les guerres fréquentes. Dans un contexte tendu de lutte entre cités, Judith devient le symbole des combats, religieux et politiques, de Florence et des Florentins.

Le message politique de l'artiste est encore difficile à établir. Botticelli se montre plus explicite dans ses peintures plus tardives. Judith devient alors une figure récurrente dans son œuvre : une Judith sortant de la tente d'Holopherne en 1495, deux représentations dans la Calomnie d'Apelle (1494-1495) ${ }^{49}$, un vaste panneau dans l'Histoire de

48 Catalogue de l'exposition Botticelli, De Laurent le Magnifique à Savonarole 2003 : 45.

49 Sandro Botticelli, La Calomnie d'Apelle, 1494-1495, peinture sur bois, 62 x $91 \mathrm{~cm}$, Florence, Musée des Offices. Deux scènes montrant Judith sont placées derrière 
Lucrèce (1496-1504) ${ }^{50}$. Dans ce dernier tableau, le lien entre la République et Judith devient évident. La République apparait en danger et un appel à une figure de justice est lancé pour la sauver. À Florence, la chute et l'exil des Médicis en 1494 conduisent à une reconversion de la statue Judith et Holopherne de Donatello. Elle devient le symbole exclusif de la République triomphante sur les tyrans Médicis. Elle est alors déplacée du palais familial au Palazzo Vecchio. Les peintures de Judith par Botticelli au cours de cette période pourraient correspondre à cette association de Judith avec la République de Florence. D'autant que Botticelli soutient la réforme de Savonarole.

Pourtant, la figure de Judith commence à déplaire à la fin du XV siècle. L'héroïne biblique évoque quand même un dangereux renversement des valeurs : une femme domine un homme et le tue. Botticelli a participé à une commission réunie par la République pour choisir le futur emplacement du David sculpté par le jeune MichelAnge. Il a ainsi approuvé le déplacement de la Judith de Donatello vers un lieu moins visible, moins public. L'héroïne du Moyen Âge serait-elle renvoyée vers la sphère privée réservée aux femmes?

\section{Bibliographie}

1998, La Bible de Jérusalem, Paris, Cerf.

2003, Botticelli, De Laurent le Magnifique à Savonarole, Paris, Skira, Musée du Luxembourg.

ANDERSON Jaynie, 1997, Judith, Paris, éditions du regard.

le trône de Midas : en haut, un panneau montrant Judith et Abra rangeant la tête d'Holopherne dans un sac; au-dessous, une statue de Judith avec à ses pieds la tête d'Holopherne. Le panneau de la tribune est difficilement identifiable : soit il s'agit d'une autre Judith en pied soit de Yael soit Artémis (la jeune femme représentée semble tenir un long instrument : un arc ?).

50 Sandro Botticelli, L'Histoire de Lucrèce, 1496-1504, détrempe et huile sur bois, 83,5 x $180 \mathrm{~cm}$, Boston, Gardner Museum. Le panneau au-dessus du viol de Lucrèce montre trois scènes : à l'arrière-plan, Béthulie assiégée par les cavaliers assyriens, au premier plan Judith et Abra rangeant la tête d'Holopherne dans un sac et Judith revenant à Béthulie. 
BARon Hans, 1955, The Crisis of the Early Italian Renaissance, Princeton, Princeton University Press.

Bialostocky Jan, 1981, «La gamba sinistra della Giuditta : il quadro di Giorgione nella storia del tema », in Rodolfo PALluchinI (ed.), Giorgione e l'umanesimo veneziano, Firenze, p. 193-226.

Blake MC Ham Sarah, 2001, "Donatello's Bronze "David" and "Judith" as Metaphors of Medici Rule in Florence», The Art Bulletin, tome 83, mars, $\mathrm{n}^{\circ} 1$, p. 32-47.

BurZLAFF Mary Caroline, 2006, Chaste sexual warrior, civic heroin and femme fatale : three views of Judith in Italian Renaissance and Baroque art, Master of Arts, University of Cincinnati.

Callahan Leslie Abend, 1998, «Ambiguity and Appropriation : The Story of Judith in Medieval Narrative and Iconographic Traditions", in Francesca CANADE Sautman, Diana Conchado \& Giuseppe Di Scipio (ed.), Telling Tales. Medieval narratives and the folk tradition, Londres, MacMillan Press, p. 79-99.

CAPOZZI Frank, 1975, The evolution and transformation of the Judith and Holofernes theme in Italian drama and art before 1627, Ph. D., The University of Wisconsin-Madison.

Casagrande Carla \& Silvana Vecchio, 2003, Histoire des péchés capitaux au Moyen Âge, Paris, Aubier. Traduction française de I sette viz̨i capitali. Storia dei peccati nel Medioevo, Turin, Einaudi, 2000.

CiletTI Elena, 1991, "Patriarchal Ideology in the Renaissance Iconography of Judith », in Marylin MigIEL \& Juliana SCHIESARI (ed.), Perspectives on Gender and the Italian Renaissance, Londres, Cornell University Press, p. 35-70.

Contamine Philippe, 1999 (5e éd. Corrigée ; 1re éd. 1980), La guerre au Moyen Âge, Paris, PUF.

Coville Alfred, 1974 (1 1 re éd. 1932), Jean Petit. La question du tyrannicide au commencement du XVe siècle, Genève, Slatkine Reprints.

Delumeau Jean-Pierre \& Isabelle Heullant-Donat, 2002, L'Italie au Moyen Âge, $V^{e}-X V^{e}$ siècle, Paris, Hachette.

DubARLE André Marie, 1966, Judith; formes et sens des diverses traditions, tome 1 : Etudes, tome 2 : Textes, Rome, Institut biblique pontifical.

Edgerton Samuel Y. Jr, 1972, «Maniera and the mannaia: decorum and decapitation in the sixteenth century ", in Franklin W. RoBINSON \& Stephen G. Nichols Jr (ed.), The meaning of mannerism, Hanover, University press of New England, , p. 67-102.

Friedman Mira, 1986, "The metamorphoses of Judith», Jewish Art Journal, 12, p. 226-246. 
GAUVARD Claude, 2001, «Honneur de femme et femme d'honneur en France à la fin du Moyen Âge », Francia, 28/1, p. 159-191.

Gervais Diane \& Serge Lusignan, 1999, «De Jeanne d'Arc à Madeleine de Verchères : la femme guerrière dans la société d'Ancien Régime », Revue d'bistoire de l'Amérique française, 53/2, p. 171-205.

JOANNIDES Paul, 1992, "Titian's Judith and its context: the iconography of decapitation », Apollo, CXXXV, p. 163-171.

Lightbown Ronald, 1990, Botticelli, Paris, Citadelles. Traduction française de Sandro Boticelli : Life and work, New York, Abbeville Press, 1989.

Migne Jacques-Paul, 1878-1891, Patrologiae latinae cursus completus, Paris, Garnier et Migne.

Smith Susan L., 1995, The power of women, a topos in medieval art and literature, Philadelphie, University of Pennsylvania Press.

TurCheTti Mario, 2001, Tyrannie et tyrannicide de l'Antiquité à nos jours, Paris, PUF.

WIND Edgar, 1985 (éd. revue et augm., 1 re éd. 1983), The eloquence of symbols, Studies in bumanist art, Oxford, Clarendon Press. 